\title{
Influencia de la sobremaduración en las propiedades mecánicas y comportamiento a corrosión bajo tensión de una aleación de aluminio 7075 T6
}

\author{
J. M. BADÍA, J. M. ANTORANZ, P. TARÍN, C. LÓPEZ DEL CASTILLO, A. G. SIMÓN, N. M. PIRIS \\ Departamento de Materiales y Producción Aeroespacial. E.T.S.I. Aeronáuticos. U.P.M. 28040 Madrid
}

\begin{abstract}
En este trabajo se estudia el efecto de la aplicación de una etapa de remaduración posterior al estado T6 sobre las propiedades mecánicas y el comportamiento a corrosión bajo tensiones de una aleación EN AW 7075. Todas las remaduraciones se dieron durante 27 horas a tres temperaturas distintas: 147,152 y $163^{\circ} \mathrm{C}$; esta última corresponde a la indicada por la norma MIL-H-6088F para la obtención del estado T73 a partir del estado T6. Se evalúan las propiedades mecánicas y a corrosión bajo tensiones de estos tratamientos, teniendo como referencia los tratamientos T6 y T73. Se analiza la evolución de la microestructura con técnicas de microscopia electrónica de transmisión (TEM) y ensayos de calorimetría diferencial de barrido (DSC) y se compara con las propiedades obtenidas.

La remaduración posterior al T6 produce una estructura más estable según aumenta la temperatura, disminuyendo la susceptibilidad a corrosión bajo tensiones. Para temperaturas inferiores a $147^{\circ} \mathrm{C}$, esta reducción no va acompañada de una disminución en las propiedades mecánicas. La remaduración a $147^{\circ} \mathrm{C}$ tiene mucho mejor comportamiento a corrosión bajo tensiones que el T6, sin deteriorar sus propiedades mecánicas. La remaduración a $152^{\circ} \mathrm{C}$ proporciona características a corrosión bajo tensiones algo peores a las del T73, con mejores propiedades mecánicas.
\end{abstract}

Palabras clave: Aleaciones de Aluminio,7075, corrosión bajo tensiones, tratamientos térmicos, microestructura, propiedades mecánicas.

\section{Influence of overaging on the mechanical properties and stress corrosion cracking behaviour of $7075 \mathrm{~T} 6$ aluminium alloy.}

The effect of a reageing heat treatment following a T6 temper on the mechanical properties and the stress corrosion cracking behaviour (SCC) on the EN AW 7075 aluminium alloy is studied. The reageing time was 27 hours for all the temperatures used: 147,152 and $163^{\circ} \mathrm{C}$; this last temperature is indicated in the MIL-H-6088F standard to obtain the T73 temper from the T6 temper. The mechanical properties and the stress corrosion behaviour are evaluated and, compared with the T6 and T73 tempers. The evolution of the microstructure is analysed with transmission electron microscopy (TEM) and differential scanning calorimetry (DSC) techniques, and related with the properties.

The reageing following to the T6 temper made a more stable structure higher the temperature, and, consequently, lowing the stress corrosion susceptibility. For temperatures lower than $147^{\circ} \mathrm{C}$, this diminution of stress corrosion susceptibility occurs without reducing the mechanical properties. So, the $147^{\circ} \mathrm{C}$ reageing heat treatment has better stress corrosion behaviour than the $\mathrm{T} 6$ temper, with similar mechanical properties. By the other hand, the stress corrosion susceptibility achieved with the $152{ }^{\circ} \mathrm{C}$ reageing is slightly worse than the T73 one, but their mechanical properties are better.

Key words: Aluminium alloys,7075, stress corrosion cracking (SCC), thermal treatments, microstructure, mechanical properties.

\section{INTRODUCCIÓN}

Las aleaciones $\mathrm{Al}-\mathrm{Zn}-\mathrm{Mg}-\mathrm{Cu}$, de gran importancia en la industria aeronáutica, se utilizan en ocasiones con tratamientos de sobremaduración (tales como el T73) para evitar el mal comportamiento a corrosión bajo tensiones que presentan en estado T6, a pesar de la disminución de características mecánicas que ello conlleva (1). Dicha pérdida de propiedades supone en muchos casos una penalización en peso de la estructura, por lo que son numerosos los intentos de desarrollar tratamientos alternativos que, manteniendo las características mecánicas del estado T6, proporcionen un mejor comportamiento a corrosión bajo tensiones $(2,3)$.

Por otro lado, la norma norteamericana MIL-H-6088F permite obtener el tratamiento T73 a partir del T6 mediante una maduración adicional o "remaduración", admitiendo un margen tanto de tiempos como de temperaturas en esa remaduración para conseguir finalmente el estado T73. En un trabajo anterior (4), se estudió la influencia de esos márgenes que la norma admite sobre las propiedades mecánicas finales y en el comportamiento a corrosión bajo tensiones de una aleación EN AW 7075 originalmente en estado T6 . En ese trabajo se observó que la temperatura de remaduración, (dentro del margen aceptado por la norma), tenía gran influencia sobre las propiedades mecánicas alcanzables (disminuyéndolas a medida que aumentaba), pero no en el comportamiento en corrosión bajo tensiones, que en todos los casos era excelente y similar al correspondiente al tratamiento T73.

En este trabajo se estudia el efecto que tiene la aplicación de una remaduración al estado T6 en función de la temperatura de tratamiento, con el objetivo de localizar posibles valores de temperatura que permitiesen mantener los niveles de propiedades mecánicas del estado T6, pero cuyo comportamiento a corrosión bajo tensiones fuese significativamente mejor. Los distintos tratamientos realizados se valoran y comparan respecto de sus características mecánicas, conductividad eléctrica, comportamiento a corrosión bajo tensiones y estructura de precipitación, tomando como referencia los valores correspondientes a los estados T6 y T73.

\section{MATERIAL Y ENSAYOS EXPERIMENTALES}

Para la realización de este trabajo se ha utilizado una plancha de $25 \mathrm{~mm}$ de espesor de la aleación comercial EN AW 7075 en estado T7351, cuya composición se indica en la tabla I. 
TABLA I.- COMPOSICIÓN PORCENTUAL EN PESO DE LA ALEACIÓN EN AW 7075 UTILIZADA

\begin{tabular}{|c|c|c|c|c|c|c|c|c|}
\hline $\mathrm{Al}$ & $\mathrm{Zn}$ & $\mathrm{Mg}$ & $\mathrm{Cu}$ & $\mathrm{Si}$ & $\mathrm{Fe}$ & $\mathrm{Cr}$ & $\mathrm{Mn}$ & $\mathrm{Ti}$ \\
\hline Base & 5.4 & 2.5 & 1.6 & 0.16 & 0.17 & 0.21 & 0.02 & 0.01 \\
\hline
\end{tabular}

Todos los tratamientos térmicos realizados para el presente trabajo (que se aplicaron a trozos de 180x160 mm cortados de una plancha de $25 \mathrm{~mm}$ de espesor) tenían una primera etapa de solución a $475^{\circ} \mathrm{C}$, seguida de enfriamiento en agua. Con posterioridad se aplicaba la etapa de maduración, de acuerdo con los valores indicados en la tabla II, de cara a conseguir, por un lado, los estados T6 y T73 de acuerdo con la normativa correspondiente, y por otro poder disponer de muestras de material a las que, tras alcanzar el estado T6, se les provocase una cierta sobremaduración mediante un nuevo calentamiento a temperatura de maduración más elevada. El tratamiento de remaduración a $163{ }^{\circ} \mathrm{C}$ durante 27 horas cumple lo especificado por la norma MIL-H-6088F para pasar de T6 a T73.

TABla II.- TRATAMIENTOS TÉRMiCOS APLiCAdOS A LA ALEACión EN AW 7075

\begin{tabular}{|c|l|}
\hline Denominación & \multicolumn{1}{|c|}{ Descripción del Tratamiento Térmico } \\
\hline $\mathrm{T} 6$ & Solución +24 horas a $120^{\circ} \mathrm{C}$ \\
$\mathrm{T} 6+147^{\circ} \mathrm{C} / 27 \mathrm{~h}$ & Solución +24 horas a $120^{\circ} \mathrm{C}+27$ horas a $147^{\circ} \mathrm{C}$ \\
$\mathrm{T} 6+152^{\circ} \mathrm{C} / 27 \mathrm{~h}$ & Solución +24 horas a $120^{\circ} \mathrm{C}+27$ horas a $152^{\circ} \mathrm{C}$ \\
$\mathrm{T} 6+163^{\circ} \mathrm{C} \not 27 \mathrm{~h}$ & Solución +24 horas a $120^{\circ} \mathrm{C}+27$ horas a $163^{\circ} \mathrm{C}$ \\
$\mathrm{T} 73$ & Solución +7 horas a $107^{\circ} \mathrm{C}+27$ horas a $163^{\circ} \mathrm{C}$ \\
\hline
\end{tabular}

La determinación de la dureza alcanzada en los distintos tratamientos se realizó mediante el ensayo Vickers, con carga aplicada de 490,3 N, de acuerdo con la norma UNE-EN ISO 6507-1.

La conductividad eléctrica alcanzada en cada uno de los estados fue medida en la superficie de las muestras tratadas mediante un equipo Sigmatest D, utilizando la técnica de corrientes inducidas.

La determinación de las características mecánicas de límite elástico y resistencia a tracción se efectuó mediante ensayos de tracción según la norma UNE EN 10002-1, sobre probetas obtenidas en dirección longitudinal de la plancha, ensayándose dos probetas por tratamiento.

Para los ensayos de calorimetría diferencial de barrido (DSC) se ha utilizado un calorímetro Perkin-Elmer DSC System 4. Estos ensayos se han realizado a una velocidad de calentamiento de la muestra de $15^{\circ} \mathrm{C} / \mathrm{min}$.

Para los ensayos de corrosión bajo tensiones se utilizaron probetas tipo DCB ("double cantilever beam") de 140×25x25 mm. La aplicación de la carga se ha realizado en la dirección del espesor de la plancha por medio de dos tornillos, de manera que la grieta de corrosión bajo tensiones se formara y creciera en un plano paralelo al de laminación. La formación de la pregrieta inicial se ha realizado por sobrecarga (popin), por lo que el $\mathrm{K}_{\mathrm{I}}$ inicial era del orden del $\mathrm{K}_{\mathrm{IC}}$ del material para cada tratamiento. A continuación las probetas eran sometidas a inmersión alternativa en una disolución acuosa de $\mathrm{NaCl}$ al $3.5 \%$, de acuerdo con la norma ASTM G-44 durante un periodo de 90 días, midiéndose periódicamente el tamaño de la grieta mediante técnicas de ultrasonidos. Para cada tratamiento térmico se han ensayado cuatro probetas DCB.

La preparación de las muestras para su observación en el microscopio electrónico de transmisión fue realizada con pulido electrolítico, utilizando una disolución de ácido nítrico en metanol al $30 \%$ a a $-30^{\circ} \mathrm{C}$. La muestras se examinaron en un equipo JEOL JEM 2010, operando a $200 \mathrm{kV}$.

\section{RESULTADOS}

Los resultados de dureza, propiedades mecánicas y conductividad eléctrica obtenidos para los diferentes tratamientos se pueden ver en la tabla III. Las mejores características se obtienen con el tratamiento T6. La posterior aplicación de un nuevo calentamiento durante 27 horas a $147^{\circ} \mathrm{C}$ no ha afectado significativamente a las propiedades mecánicas de la aleación, alcanzándose valores análogos a los del T6. Por el contrario, los calentamientos realizados a 152 y $163{ }^{\circ} \mathrm{C}$ han provocado una disminución de características tanto mayor cuanto mayor ha sido la temperatura, de manera que tras la permanencia a $163^{\circ} \mathrm{C}$ se obtienen resultados similares (aunque algo mayores) a los conseguidos tras el tratamiento T73.

TABLA III.- CONDUCTIVIDAD ELÉCTRICA Y PROPIEDADES MECÁNICAS OBTENIDAS CON LOS TRATAMIENTOS TÉRMICOS APLICADOS

\begin{tabular}{|l|l|l|l|l|}
\hline Tratamiento & $\begin{array}{l}\text { Conductividad } \\
M S / m\end{array}$ & $\begin{array}{l}\text { Dureza } \\
\text { HV50 }\end{array}$ & $\begin{array}{l}\text { Limite Elástico } \\
R_{p 0,2^{\prime}} M P a\end{array}$ & $\begin{array}{l}\text { Resistencia } \\
R_{m^{\prime}} M P a\end{array}$ \\
\hline $\mathrm{T} 6$ & 17.9 & 195 & 536 & 601 \\
\hline $\mathrm{T} 6+147^{\circ} \mathrm{C} / 27 \mathrm{~h}$ & 19.4 & 189 & 544 & 597 \\
\hline $\mathrm{T} 6+152^{\circ} \mathrm{C} / 27 \mathrm{~h}$ & 20.2 & 183 & 528 & 587 \\
\hline $\mathrm{T} 6+163^{\circ} \mathrm{C} / 27 \mathrm{~h}$ & 21.8 & 173 & 476 & 541 \\
\hline $\mathrm{T} 73$ & 22.1 & 170 & 465 & 534 \\
\hline
\end{tabular}

Por otro lado, como era de esperar, el tratamiento que proporciona la menor conductividad eléctrica es el T6. La aplicación posterior de una nueva remaduración a más temperatura produce un aumento de la conductividad eléctrica tanto mayor cuanto más elevada es dicha temperatura. Tras la remaduración a $163^{\circ} \mathrm{C}$ se alcanza un valor próximo al correspondiente al estado T73.

Los termogramas obtenidos en DSC con los distintos tratamientos térmicos se han agrupado en la figura 1, en la que se han decalado las diferentes curvas para facilitar su observación.

El tratamiento T6 presenta un primer pico endotérmico, entre 150 y $230{ }^{\circ} \mathrm{C}$, que se transforma bruscamente en una sucesión de picos exotérmicos solapados, terminando la curva con una amplia zona endotérmica con final hacia $\operatorname{los} 460^{\circ} \mathrm{C}$. La aplicación posterior de una remaduración va haciendo ensancharse al pico endotérmico inicial, a la vez que su máximo se desplaza a temperaturas superiores. Los picos exotérmicos que aparecen claramente en el ensayo de T6 se reducen drásticamente hasta desaparecer por completo tras la remaduración a $152{ }^{\circ} \mathrm{C}$. Por último, la zona endotérmica final no se ve significativa-

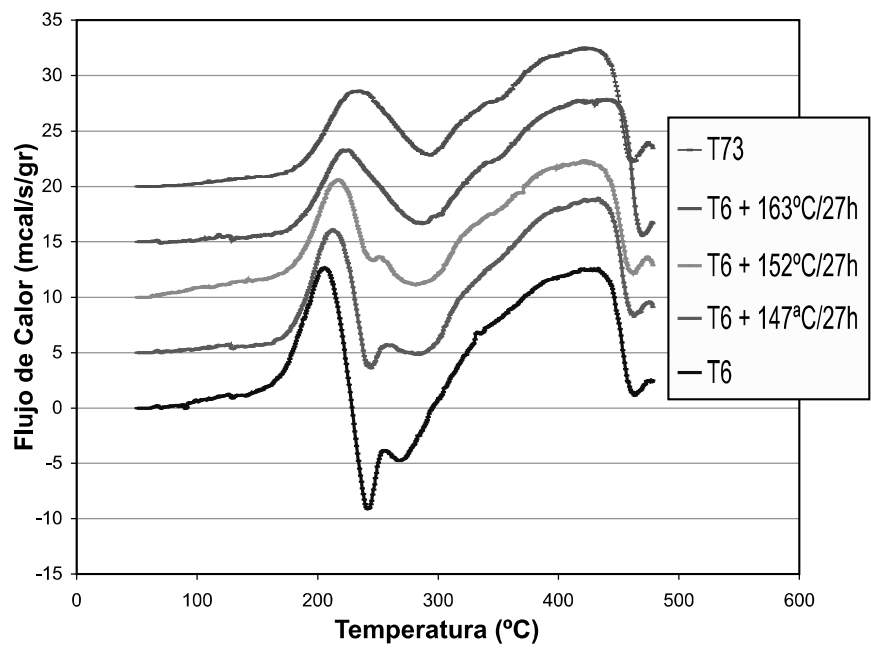

Figura 1.- Curvas DSC a $15^{\circ} \mathrm{C} / \mathrm{min}$, de los tratamientos térmicos realizados 
mente afectada por la aplicación de las remaduraciones. Viendo de forma global del conjunto de gráficos, queda claro que la aplicación de dichas remaduraciones va haciendo evolucionar el termograma, y en consecuencia la estructura de precipitación obtenida durante el tratamiento térmico, hacia el estado T73, de manera que las curvas correspondientes a este estado y la de la remaduración a $163{ }^{\circ} \mathrm{C}$ son similares.

En la figura 2, se pueden ver las curvas de crecimiento de grieta en función del tiempo durante los ensayos de corrosión bajo tensiones con los distintos tratamientos realizados. Cada una de ellas recoge los valores medios obtenidos en las cuatro probetas ensayadas para cada tratamiento. La curva correspondiente al tratamiento T6 muestra un rápido crecimiento de la grieta, que se mantiene hasta prácticamente la rotura total de la probeta. La remaduración a $147^{\circ} \mathrm{C}$ provoca que exista un corto periodo inicial durante el cual no se aprecia un crecimiento de grieta; además la grieta crece con un ritmo mucho menor que con el tratamiento T6. La curva de las muestras remaduradas a $152{ }^{\circ} \mathrm{C}$ sigue estas mismas tendencias pero más acentuadas (mayor retraso inicial del crecimiento de la grieta y menor crecimiento de grieta). Finalmente, la remaduración a $163^{\circ} \mathrm{C}$ y el tratamiento T73 tienen un comportamiento similar en corrosión bajo tensiones: la grieta prácticamente no crece con estos tratamientos.

En la figura 3 se presentan las valores de la velocidad de crecimiento de grieta en función del factor de intensidad de tensiones $K_{1}$ existente en el frente de grieta. Los resultados obtenidos para los tratamientos T6 y T73 concuerdan plenamente con estudios anteriores (5). En dicha figura se ve una disminución de la velocidad de crecimiento de grieta al aumentar la temperatura de remaduración, llegándose, para la remaduración a $163^{\circ} \mathrm{C}$ a un comportamiento totalmente análogo al que presenta el T73. Las remaduraciones a las temperaturas intermedias también reducen de forma muy significativa la velocidad de crecimiento respecto del $\mathrm{T} 6$.

El microscopio electrónico de transmisión ha sido utilizado para el estudio de la estructura de precipitación. En la figura 4 se muestran microestructuras correspondientes a los distintos tratamientos realizados, en la que se han escogido campos en los que se aprecie, preferentemente, la estructura del borde de grano. Los precipitados de borde grano muestran un ligero aumento de tamaño con la temperatura de remaduración (Fig. 4a-4c), pero sin llegar a alcanzar el tamaño de los precipitados obtenidos con el tratamiento T73 (Fig. 4d); la zona libre de precipitados (PFZ) alrededor de los límites de grano también crece ligeramente con la temperatura de remaduración, pero tampoco se acerca a la que tiene en el T73. La estructura en el interior de los granos muestra, asimismo, la misma tendencia que el borde de grano, es decir, un aumento del tamaño de los precipitados con la temperatura de remaduración.

\section{DISCUSIÓN}

La remaduración posterior al tratamiento T6 provoca una estabilización de la estructura, tanto mayor cuanto más elevada es la temperatura, como confirma el aumento continuo de la conductividad (Tabla III) y la evolución de los termogramas de DSC (Fig. 1). Esta evolución hacia la estabilidad de la estructura de precipitación normalmente lleva asociada una disminución de las propiedades mecánicas, pero en esta ocasión la remaduración a $147^{\circ} \mathrm{C}$ no ha producido una variación significativa de éstas. Para mayores temperaturas sí que aparece una pérdida progresiva.

Estas variaciones de propiedades mecánicas y conductividad se deben a los cambios de tipo y tamaño de precipitados presentes en la estructura.

Los ensayos de DSC ayudan a explicar estos cambios (Fig. 1). La estructura en el estado T6 ha sido ampliamente estudiada, aunque todavía se mantienen importantes diferencias de opinión acerca de su constitución. El primer pico endotérmico en torno a $200{ }^{\circ} \mathrm{C}$ ha sido identificado en estudios previos como de disolución de precipitados mayoritariamente semicoherentes (6-8); no obstante, algunos autores opinan que la fase mayoritaria estaría constituida fundamentalmente por zonas coherentes "GP" $(9,10)$. El complejo pico exotérmico que aparece entre $240{ }^{\circ} \mathrm{C}$ y $300{ }^{\circ} \mathrm{C}$ correspondería a la formación de fases incoherentes estables.

El hecho de que el pico endotérmico inicial se vaya desplazando hacia temperaturas superiores y a la vez ensanchándose, corresponde a que la estructura de precipitación desarrollada durante el tratamiento es cada vez más estable, es decir, los precipitados van siendo cada vez más incoherentes con la matriz y de mayor tamaño, por lo que interaccionan menos con las dislocaciones y en consecuencia disminuyen las características mecánicas y aumenta la conductividad eléctrica.

El comportamiento en corrosión bajo tensiones se ve mejorado con

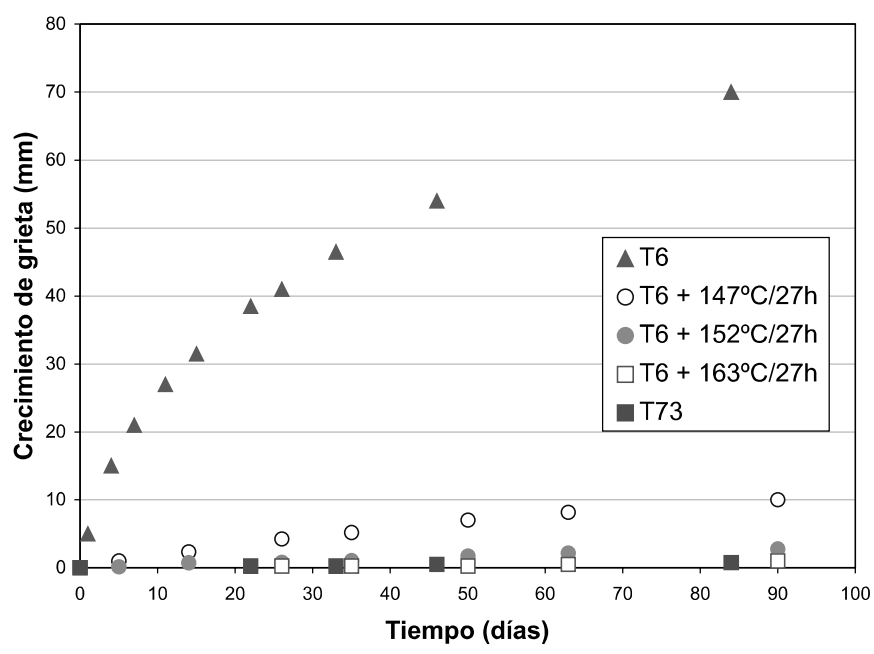

Figura 2.- Crecimiento de grieta por corrosión bajo tensiones en probeta $\mathrm{DCB}$, para los tratamientos térmicos realizados

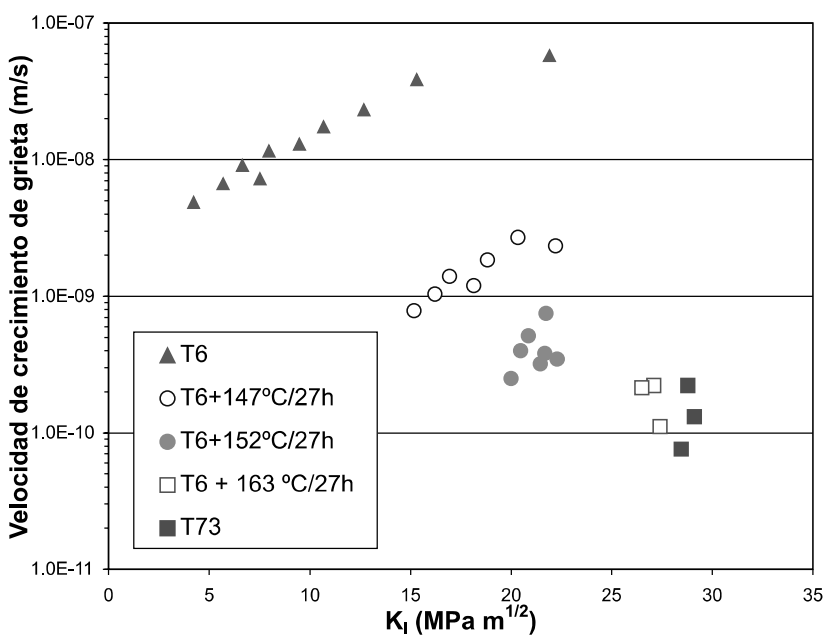

Figura 3.- Velocidad de crecimiento de grieta en función del factor de intensidad de tensiones para los tratamientos estudiados 

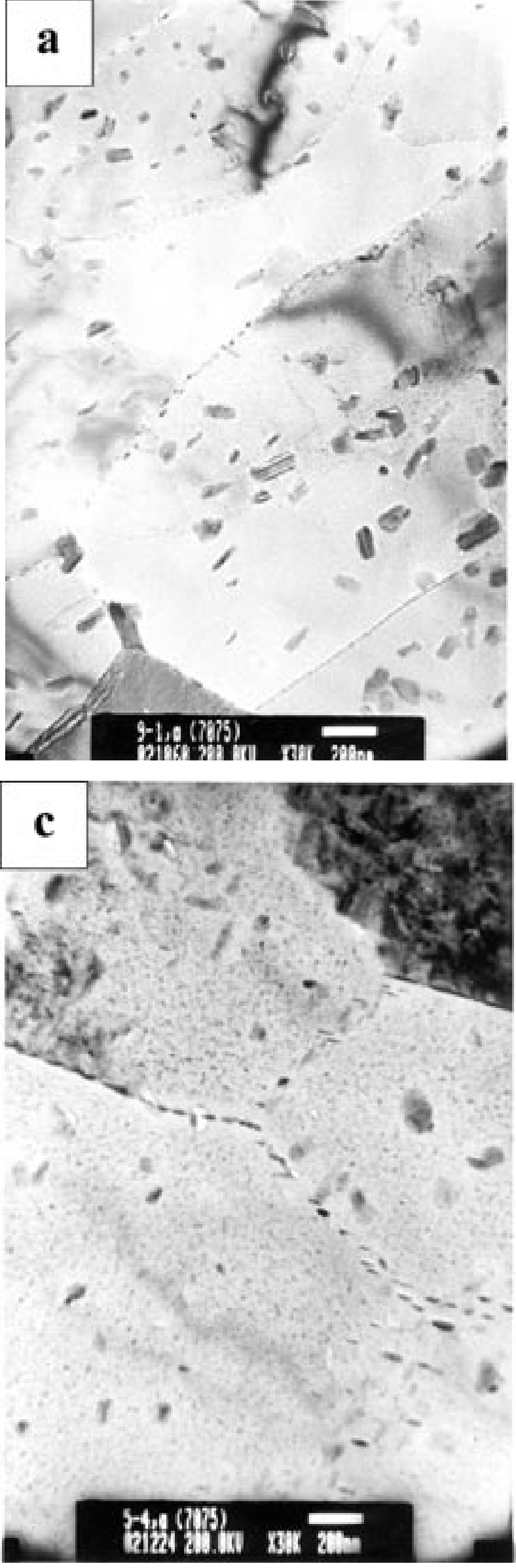
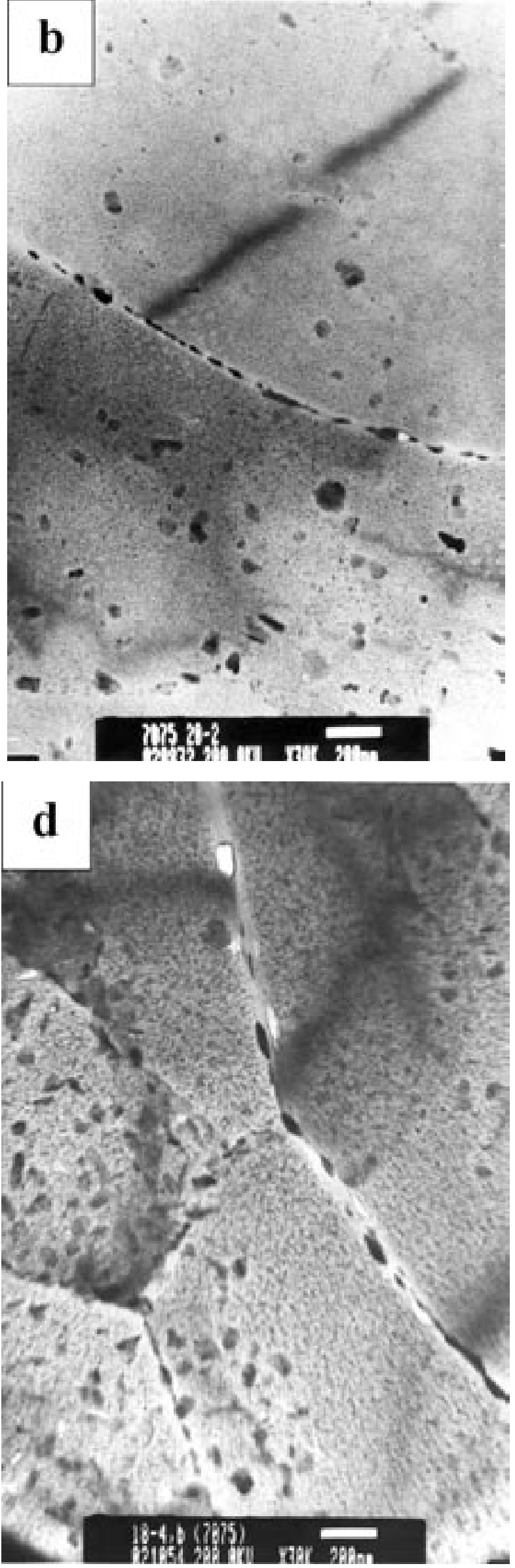

Figura 4.-Micrografías obtenidas por microscopia electrónica de transmisión para los tratamientos a) T6, b) T6 + $147^{\circ} \mathrm{C} / 27 \mathrm{~h}$, c) $\mathrm{T} 6$ + $163{ }^{\circ} \mathrm{C} / 27 \mathrm{~h}$, d) $\mathrm{T} 73$. 
la remaduración con cualquiera de las temperaturas ensayadas (Fig. 2). Se han realizado numerosos estudios $(7,10,11)$ en los que se correlaciona microestructura y comportamiento en corrosión bajo tensiones, sin que se haya encontrado una única característica microestructural que explique ese comportamiento, siendo lo más habitual considerarlo asociado a la microestructura en el borde de grano: tamaño de los precipitados y anchura de la zona libre de precipitados (PFZ). Comparando las micrografias de los tratamientos T6 y T73 (Fig.4a, 4d), se aprecia claramente un aumento tanto del tamaño de los precipitados, como de las PFZ, lo cual debe ser relacionado con la gran disminución (dos órdenes de magnitud) de la velocidad de crecimiento de grieta del T73 con respecto al T6 (Fig. 3).

En los tratamientos con remaduración se producen variaciones evidentes en la velocidad de propagación de la grieta, mientras que los cambios en la microestructura son mucho menos claros, aún comparándolos con la estructura del T6.

Por otro lado, las estructuras de los tratamientos T73 (Fig. 4d) y la remaduración a $163^{\circ} \mathrm{C}$ (Fig. 4c) son diferentes, pero el comportamiento en corrosión bajo tensiones es prácticamente igual, lo que indica que no se puede deducir el comportamiento a corrosión de la aleación únicamente mediante el estudio de la estructura de precipitación de borde de grano.

\section{CONCLUSIONES}

La remaduración posterior al estado T6 estabiliza progresivamente la estructura de la aleación, modificando el tipo de precipitados y aumentando el tamaño de los mismos. Esta estabilización de la estructura produce una mejora sustancial en el comportamiento a corrosión bajo tensiones para todas las temperaturas estudiadas, y una disminución en las propiedades mecánicas, pero sólo a partir de una temperatura. En la remaduración a $147^{\circ} \mathrm{C}$ se produce una notable mejora del comportamiento a corrosión, sin pérdida apreciable de propiedades mecánicas, con respecto al T6.

Como se puede ver, la mejora en el comportamiento a corrosión no tiene por qué llevar asociado, necesariamente, un deterioro en las propiedades mecánicas, ya que se ha podido conseguir con una remaduración adecuada la mejora en corrosión sin que todavía sea apreciable la caída en las características de tracción, lo cual abre paso al posible desarrollo industrial de tratamientos térmicos alternativos y fáciles de aplicar que reduzcan la susceptibilidad a corrosión bajo tensiones sin perjudicar el comportamiento mecánico de la aleación.

\section{AGRADECIMIENTOS}

Los autores agradecen al Laboratorio de Microscopia Electrónica del Centro Nacional de Investigaciones Metalúrgicas (CENIM), y en particular a la Dra. Paloma Adeva, el apoyo prestado para los ensayos de microscopia.

\section{BIBLIOGRAFÍA}

1. I. J. Polmear. Ligth Alloys, ed. Edward Arnold Publishers Ltd., Londres, 1981.

2. E Di Russo et al.. "Thermomechanical treatments on high strength Al-ZnMg-(Cu), Met. Trans. 4, 1133-1144, (1973).

3. B. M. Cina. "Reducing the susceptibility of alloys, particularly aluminium alloys, to stress corrosion cracking," U. S. Patent 3,856,584, December 24, 1974.

4. J. Ma . Badía, J. M. Antoranz, N. Martín, A. García, C. López Del Castillo, I. Fernández. "Optimización del tratamiento térmico aplicable a las aleaciones Al-Zn-Mg-Cu para conseguir el estado T73 a partir del estado T6", pp451, $7^{\circ}$ Congreso Nacional de Tratamientos Térmicos y de Superficie, Madrid (España), Mayo 1998. Ed. Centro Nacional de Investigaciones Metalúrgicas, Madrid, 1998.

5. M. O. Speidel. "Stress corrosion cracking of aluminium alloys". Met. Trans. Vol. 6A, 631-651, April 1975.

6. J. Mª Badía, R. Servent, C. López del Castillo, J. M. Antoranz. “Influencia de la modificación del proceso de temple sobre la precipitación en la aleación de aluminio 7075 (L-3710)", 187-196, Congreso Nacional de Ciencia y Tecnología Metalúrgicas, Asamblea General de CENIM, 3-5 Octubre 1990.

7. J. K. Park, A. Ardell. "Effect of retrogression and reaging treatments on the microstructure of Al 7075-T651", Metals Technology, 10,386-392, (1983).

8. J. K. Park. "Influence of retrogression and reaging treatments on the strength and stress corrosion cracking of aluminium alloy 7075-T6", Mat. Sci. Eng. A103, 223-231, (1988).

9. M. U. Islam, W. Wallace. "Retrogression and reaging response of 7475 aluminium alloy", Metals Technology, 10, 386-392, (1983).

10. K. Rajan, W. Wallace, I. C. Beddoes. "Microstructural study of a high strength stress corrosion resistant 7075 aluminium alloy", J. Mat. Sci., 17, 2817-2824, (1982).

11. A. Uguz, J. W. Martín, “The measurement of grain boundary particle distributions in aged Al-Zn-Mg alloys", Materials Characterization, 27, 147-156, (1991).

Recibido: 1.2.03

Aceptado: 30.11 .03 